\title{
The first space-based gravitational-wave detectors
}

\author{
R. R. Caldwell* \\ Department of Physics and Astronomy, University of Pennsylvania, 209 South 33rd Street, Philadelphia, Pennsylvania 19104 \\ Marc Kamionkowski ${ }^{\dagger}$ and Leven Wadley \\ Department of Physics, Columbia University, 538 West 120th Street, New York, New York 10027
}

(Received 28 July 1998; published 24 December 1998)

\begin{abstract}
Gravitational waves provide a laboratory for general relativity and a window to energetic astrophysical phenomena invisible with electromagnetic radiation. Several terrestrial detectors are currently under construction, and a space-based interferometer is envisioned for launch early next century to detect test-mass motions induced by waves of relatively short wavelength. Very-long-wavelength gravitational waves can be detected using the plasma in the early Universe as test masses; the motion induced in the plasma by a wave is imprinted onto the cosmic microwave background $(\mathrm{CMB})$. While the signature of gravitational waves on the CMB temperature fluctuations is not unique, the polarization pattern can be used to unambiguously detect gravitational radiation. Thus, forthcoming CMB polarization experiments, such as the Microwave Anisotropy Probe and Planck, will be the first space-based gravitational-wave detectors. [S0556-2821(99)01002-4]
\end{abstract}

PACS number(s): 95.55.Ym, 04.80.Nn, 98.70.Vc

One of the most spectacular predictions of general relativity is the existence of gravitational waves. A gravitational wave conveys information about the motions of mass and ripples in curvature-the shape of spacetime. Detection of gravitational radiation would allow us to probe "invisible", astrophysical phenomena hidden from view by absorption of electromagnetic radiation. Observations of the binary pulsar PSR1913+16, which confirm the orbital-inspiral rate due to the emission of gravitational waves [1], bring us tantalizingly close to this goal. However, we would still like to detect gravitational radiation directly. Thus, a variety of efforts are now under way to detect gravitational waves [2]. Here, we show that forthcoming maps of the polarization of the cosmic microwave background (CMB) can be used to detect very-long-wavelength gravitational radiation.

Gravitational waves are detected by observing the motion they induce in test masses $[3,4]$. High-frequency $\left(1-10^{4} \mathrm{~Hz}\right)$ gravitational waves, produced by the inspiral and catastrophic collision of astrophysical objects, may be detectable by terrestrial laser interferometers [e.g., the Laser Interferometric Gravitational Wave Observatory (LIGO) [5]] or resonant-mass antennae currently under construction. Low-frequency $\left(10^{-4}-10^{-1} \mathrm{~Hz}\right)$ gravitational waves, produced by the orbital motion of binaries, could be detected by the Laser Interferometer Space Antenna (LISA) [6], a spacebased interferometer targeted for launch circa 2015.

How can one detect ultra-low-frequency $\left(10^{-15}\right.$ $-10^{-18} \mathrm{~Hz}$ ) gravitational radiation, with wavelengths comparable to the size of the observable Universe? The photonbaryon fluid in the early Universe acts as a set of test masses for such waves. A gravitational wave in this frequency range alternately squeezes and stretches the primordial plasma. Just as a resonant-mass detector is equipped with electronics to

\footnotetext{
*Electronic address: caldwell@dept.physics.upenn.edu

${ }^{\dagger}$ Electronic address: kamion@phys.columbia.edu

\#Electronic address: leven@phys.columbia.edu
}

monitor the oscillation modes of the test body, the CMB photons are the electromagnetic signal upon which these plasma motions are imprinted.

One might despair because cosmological density perturbations generate equivalent fluctuations in the CMB temperature, nullifying the possibility of using the temperature fluctuations to detect gravitational waves (although it can be used to place upper limits [7]). To illustrate, we show two simulated CMB temperature and polarization maps in Fig. 1. The color contrasts represent temperature fluctuations of roughly one part in $10^{5}$ (red are hot spots and blue are cold). In Fig. 1(a), the temperature fluctuations are produced by a spectrum of stochastic cosmological density perturbations that may be expected in a realistic inflationary cosmological scenario. In Fig. 1(b) we have found a plausible spectrum of stochastic long-wavelength gravitational waves that produce precisely the same temperature fluctuations.

Yet our hopes are restored upon realization that the motions in the cosmological fluid generated by gravitational waves polarize the $\mathrm{CMB}$ in a pattern that is distinct from that produced by density perturbations [8,9]. Roughly speaking, the polarization "vector" field $\vec{P}(\hat{\mathbf{n}})$ (as a function of position $\hat{\mathbf{n}}$ on the sky) can be decomposed into a curl and curlfree part,

$$
\vec{P}(\hat{\mathbf{n}})=\vec{\nabla} A+\vec{\nabla} \times \vec{B}
$$

where $A$ is a scalar function and $\vec{B}$ is a vector field. The curl and curl-free parts of $\vec{P}(\hat{\mathbf{n}})$ can be isolated by taking the curl and gradient of $\vec{P}$, respectively. Since density perturbations are scalar perturbations to the spacetime metric, they have no handedness and therefore produce no curl in the CMB polarization field. Gravitational waves, however, do have a handedness, so they do produce a curl. By decomposing the CMB polarization field into its curl and curl-free parts, one can unambiguously detect gravitational waves. Again, to illustrate, the headless arrows in Fig. 1 depict the orientation and 


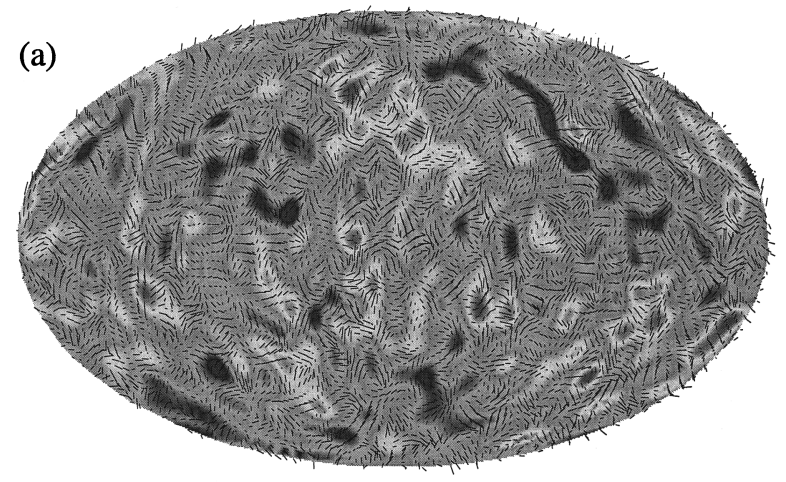

tor $\mathbf{k}$ oriented in the $\hat{\mathbf{z}}$ direction. The curl component of the polarization pattern induced by this wave will have expansion coefficients

$$
\begin{aligned}
a_{(\ell m)}^{\mathrm{C},+}(k, h)= & -\left(2 \pi^{2} i\right) h \sqrt{2 \ell+1}\left(\delta_{m, 2}-\delta_{m,-2}\right) \\
& \times \Delta_{\mathrm{C} \ell}^{(\mathrm{T})}(k, h=1) .
\end{aligned}
$$

The functions $\Delta_{\mathrm{C} /}^{(\mathrm{T})}(k, h=1)$ describe the perturbations to an isotropic photon distribution induced by a gravitational wave of initially unit amplitude $(h=1)$; they are obtained from equations for the photon distribution in an expanding Universe with this gravitational wave. The precise form of $\Delta_{\mathrm{C} /}^{(\mathrm{T})}(k, h=1)$ is only weakly dependent on the cosmological model. Only $m= \pm 2$ modes contribute since the effect of the gravitational wave is symmetric under a $180^{\circ}$ rotation about the direction of propagation.

Figure 2 shows the curl component of the polarization (as well as the temperature fluctuation) produced by such a gravitational wave, a record of the effect of the wave on the spherical surface of last scatter. The direction of propagation as well as the polarization are clearly visible in this pattern; the wavelength and the phase can also be inferred. In this regard, the CMB resembles a spherical resonant-mass detector [11] more than it resembles LISA or LIGO.

What is the smallest dimensionless amplitude $h$ of a

FIG. 1. Simulated temperature-polarization maps of the CMB sky for (a) density perturbations and (b) long-wavelength gravitational waves. Notice that the temperature anisotropy maps, indicated by the color patterns, are identical, e.g., the same cold, blue patches occur in both (a) and (b). However, the polarization patterns, indicated by the headless arrows, are distinct. For example, the orientation of the polarization vector along the border of the projection maps differs in (a) and (b). (A color version of this figure is available at $\mathrm{http}: / / \mathrm{xxx}$.lanl.gov/abs/astro-ph/9807319.)

magnitude of the polarization at each point on the sky. We see that density perturbations and gravitational waves that produce identical temperature maps can be distinguished by the polarization pattern.

More precisely, the Stokes parameters $Q(\hat{\mathbf{n}})$ and $U(\hat{\mathbf{n}})$, as a function of direction $\hat{\mathbf{n}}=(\theta, \phi)$ on the sky, are components of a symmetric trace-free (STF) $2 \times 2$ tensor,

$$
\mathcal{P}_{a b}(\hat{\mathbf{n}})=\frac{1}{2}\left(\begin{array}{cc}
Q(\hat{\mathbf{n}}) & -U(\hat{\mathbf{n}}) \sin \theta \\
-U(\hat{\mathbf{n}}) \sin \theta & -Q(\hat{\mathbf{n}}) \sin ^{2} \theta
\end{array}\right),
$$

in spherical polar coordinates, with metric $g_{a b}$ $=\operatorname{diag}\left(1, \sin ^{2} \theta\right)$. The polarization tensor can be expanded

$$
\frac{\mathcal{P}_{a b}(\hat{\mathbf{n}})}{T_{0}}=\sum_{\ell=2}^{\infty} \sum_{m=-\ell}^{\ell}\left[a_{(\ell m)}^{\mathrm{G}} Y_{(\ell m) a b}^{\mathrm{G}}(\hat{\mathbf{n}})+a_{(\ell m)}^{\mathrm{C}} Y_{(\ell m) a b}^{\mathrm{C}}(\hat{\mathbf{n}})\right],
$$

in terms of a basis $Y_{(\ell m) a b}^{\mathrm{G}}$, for the "gradient" (or "curlfree"), and $Y_{(\ell m) a b}^{\mathrm{C}}$, for the "curl," components of a STF $2 \times 2$ tensor field, and $a_{(\ell m)}^{\mathrm{G}}$ and $a_{(\ell m)}^{\mathrm{C}}$ are expansion coefficients [10].

Consider a single gravitational wave with amplitude $h$ in the early Universe, + polarization, and comoving wave vecgravitational wave of frequency $f$ that can be detected with such an experiment? Suppose a CMB polarization experiment measures $Q_{i}$ and $U_{i}$ at each of $i=1,2, \ldots, N$ small regions on the sky, each of area $4 \pi / N$. Then we have $2 N$ independent measurements of $h$, one for each $Q_{i}$ and $U_{i}$, each with an instrumental noise $\sigma$. The minimum-variance estimator of $h$ for the map is obtained from the weighted average of all of these measurements, and the variance with which $h$ can be determined is therefore $\sigma_{h}$, given by

$$
\begin{aligned}
\left(\frac{h}{\sigma_{h}}\right)^{2} & =\sum_{i} \frac{\left[Q_{i}(h=1)\right]^{2}+\left[U_{i}(h=1)\right]^{2}}{\sigma^{2}} \\
& =2 \frac{N}{4 \pi \sigma^{2}} \sum_{\ell m}\left|a_{(\ell m)}^{\mathrm{C}}\right|^{2} .
\end{aligned}
$$

In Fig. 3, we plot the results for the smallest amplitudes that can be detected at the $2 \sigma$ level by the Microwave Anisotropy Probe (MAP) [12], and the Planck Surveyor [13], CMB satellite experiments scheduled for launch, respectively, by NASA in the year 2000 and by the European Space Agency five years later. The amplitude detectable by a given CMB experiment is proportional to the instrumental noise $\sigma$ in the detector. If we extrapolate the rate of progress in detector technology the past few decades - roughly an order of magnitude per decade-into the future, then it is plausible that the sensitivity could be improved by a factor of 100 over that of Planck within the timescale for flight of LISA. Thus, we also show in Fig. 3 the smallest amplitude $h$ that could be detected by such a future $\mathrm{CMB}$ polarization experiment. The smallest $h$ detectable by LIGO and LISA are also plotted. For reference, we show the amplitude of the largest scaleinvariant stochastic gravitational-wave background consis- 


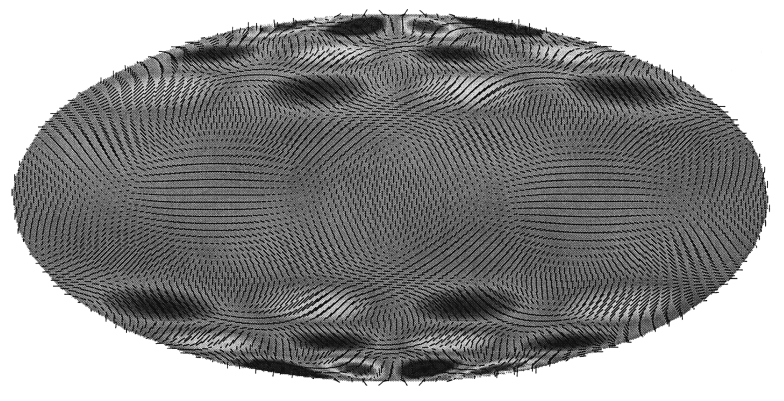

FIG. 2. The temperature and curl component of the CMB polarization pattern produced by a single gravitational wave with wave number $k=4 H_{0} / c$, where $H_{0}$ is the Hubble constant, propagating in the $\hat{\mathbf{z}}$ direction with + polarization. Density perturbations generate no curl polarization. Hence, such a pattern is a unique signal of gravitational waves. (A color version of this figure is available at http://xxx.lanl.gov/abs/astro-ph/9807319.)

tent with the Cosmic Background Explorer (COBE) [7] (the short-dashed curve) and an upper limit from pulsar timing [14] (the triangle).

Are there any promising sources of such long-wavelength gravitational radiation [15]? And if so, what could we learn from them? The most encouraging and intriguing source is the spectrum of gravitational waves produced from quantum fluctuations in the spacetime metric (analogous to Hawking radiation) during slow-roll inflation. If detected, these waves would allow us to probe the early Universe well beyond the epoch when it became opaque to electromagnetic radiation at $z \simeq 1100$, out to the inflationary epoch at redshifts $z \sim 10^{28}$, roughly $10^{-20}$ seconds after the big bang. It can also be shown that the amplitude of the stochastic gravitational-wave background depends on the energy scale of inflation. If inflation has something to do with grand unification, as many theorists surmise, then the amplitude should be $h \sim 10^{-5}$, possibly within reach of these CMB experiments. Other possible sources of ultra-low-frequency gravitational waves include bubble collisions during a first-order phase transition in the early Universe [16] or the action of topological defects [17]. Thermal gravitational waves might be left over from the Planck era [18], and string theory-inspired alternatives and/or extensions to inflation predict a unique spectrum of primordial gravitational radiation $[19,20]$.

The primary stated goals of MAP and Planck will be to determine the geometry of the Universe and the origin of

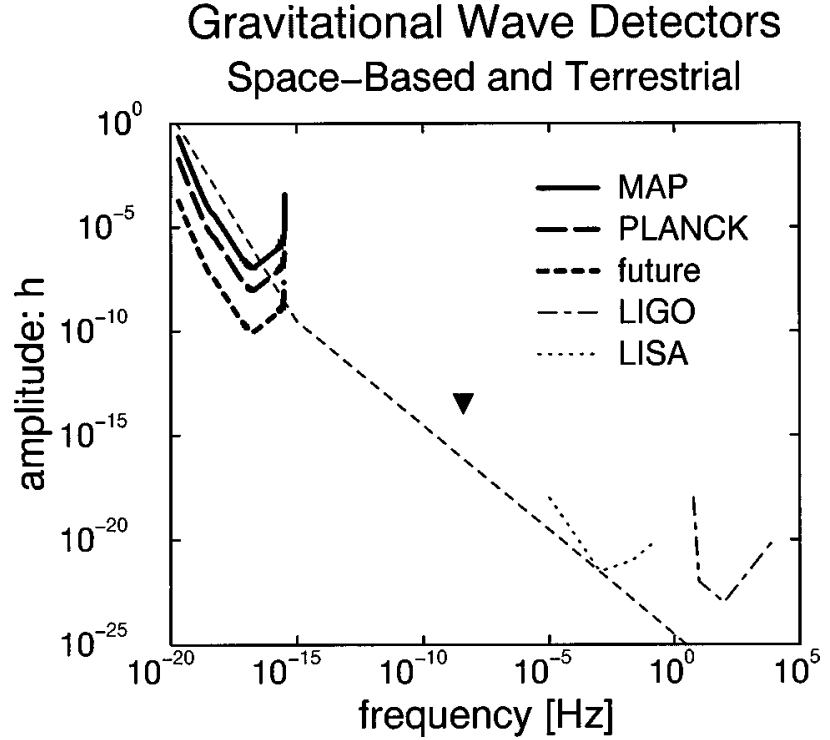

FIG. 3. The smallest amplitude $h$ of a gravitational wave that can be detected at a frequency $f$ for LIGO, LISA, MAP, Planck, and a putative future CMB polarization experiment with 100 times the Planck sensitivity. The short-dashed line shows the largest scale-invariant stochastic gravitational-wave background consistent with COBE, and the triangle shows an upper limit from pulsar timing.

large-scale structure. But as we have shown here, these satellite experiments, which will precede LISA by 10 to 20 years, may also provide the first direct detection of gravitational radiation. The primordial plasma will provide the test masses for this detector, and these gravitational-waveinduced motions are isolated unambiguously in the CMB polarization. Just as the early Universe is the poor man's particle accelerator, the CMB polarization will be the poor man's gravitational-wave detector. If these experiments register a positive result, the implications for general relativity, early-universe cosmology, and quantum theory in curved spacetime will be staggering.

This work was supported at Columbia University by the U.S. DOE contract DEFG02-92-ER 40699, NASA ATP grant NAG5-3091, and the Alfred P. Sloan Foundation, and at the University of Pennsylvania by U.S. DOE contract DEFG02-95-ER 40893.
[1] J. H. Taylor, Rev. Mod. Phys. 66, 711 (1994).

[2] E.g., K. Thorne, in Particle and Nuclear Astrophysics and Cosmology in the Next Millennium, Proceedings of the 1994 Snowmass Summer Study, edited E. W. Kolb and R. D. Peccei (World Scientific, Singapore, 1995).

[3] H. Bondi, Nature (London) 179, 1072 (1957).

[4] C. W. Misner, K. S. Thorne, and J. A. Wheeler, Gravitation (Freeman, San Francisco, 1973), pp. 444-455.

[5] A. Abramovici et al., Science 256, 325 (1992).
[6] K. Danzmann et al., "Laser Interferometer Space Antenna: Pre-Phase A Report,"' 1996.

[7] R. L. Davis et al., Phys. Rev. Lett. 69, 1856 (1992); L. M. Krauss and M. White, ibid. 69, 869 (1992).

[8] M. Kamionkowski, A. Kosowsky, and A. Stebbins, Phys. Rev. Lett. 78, 2058 (1997).

[9] U. Seljak and M. Zaldarriaga, Phys. Rev. Lett. 78, 2054 (1997).

[10] M. Kamionkowski, A. Kosowsky, and A. Stebbins, Phys. Rev. 
D 55, 7368 (1997).

[11] W. W. Johnson and S. M. Merkowitz, Phys. Rev. Lett. 70, 2367 (1993).

[12] http://map.gsfc.nasa.gov.

[13] http://astro.estec.esa.nl/SA-general/ Projects/Planck/

[14] V. M. Kaspi, J. H. Taylor, and M. F. Ryba, Astrophys. J. 428, 713 (1994).

[15] B. Allen, in Proceedings of the Les Houches School on Astrophysical Sources of Gravitational Radiation, edited by J.-A.
Marck and J.-P. Lasota (Cambridge University Press, Cambridge, England, 1997).

[16] E.g., C. Baccigalupi et al., Phys. Rev. D 56, 4610 (1997).

[17] R. R. Caldwell, R. A. Battye, and E. P. S. Shellard, Phys. Rev. D 54, 7146 (1996); X. Martin and A. Vilenkin, Phys. Rev. Lett. 77, 2879 (1996).

[18] L. P. Grischuk, JETP 40, 409 (1974).

[19] G. Veneziano, hep-th/9802057.

[20] Massimo Galluccio, Marco Litterio, and Franco Occhionero, Phys. Rev. Lett. 79, 970 (1997). 